\title{
Retraction Note to: miR-124 Acts as a Tumor Suppressor in Glioblastoma via the Inhibition of Signal Transducer and Activator of Transcription 3
}

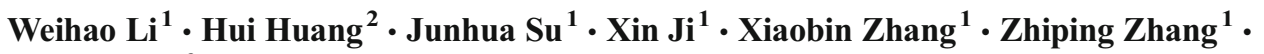 \\ Hong Wang ${ }^{3}$
}

Published online: 13 July 2017

(C) Springer Science+Business Media, LLC 2017

Retraction Note to: Mol Neurobiol (2017) 54:2555-2561

DOI 10.1007/s12035-016-9852-Z

This article has been retracted at the request of the Editorin-Chief and the Publisher per the Committee on Publication Ethics guidelines. The article shows evidence of irregularities in authorship during the submission process, there is strong reason to believe that the peer review process was compromised and the authors have plagiarized parts from the following article:

Zhen Li, Hua Wang, Zhiqiang Wang, Hongbing Cai, MiR195 inhibits the proliferation of human cervical cancer cells by directly targeting cyclin D1, Tumor Biol. (2016) 37:64576463, DOI 10.1007/s13277-015-4540-6; Received: 4 November 2015

As such the validity of the content of this article cannot be verified.

The online version of the original article can be found at http://dx.doi.org/ $10.1007 / \mathrm{s} 12035-016-9852-\mathrm{Z}$

Weihao Li

lwhaohebei@163.com

1 Department of Laboratory Medicine, Second Hospital of Hebei Medical University, No. 215 Hepingxi Road,

Shijiazhuang, Hebei 050000, China

2 Department of Laboratory Medicine, Children's Hospital of Hebei Province, Shijiazhuang, Hebei, China

3 Department of Blood Transfusion, Hebei Provincial Chest Hospital, Shijiazhuang, Hebei, China 